\title{
AN ALTERNATIVE DECOMPOSITION OF CATALAN NUMBER *
}

\author{
Predrag V. Krtolica, Predrag S. Stanimirović and Igor Stojanović
}

\begin{abstract}
A particular integer sequence derived by the convex polygon triangulation is introduced and investigated. After some underlying results are presented, the forbidden (or improper) integer values relative to the triangulation are concerned. It is understood that the forbidden sequences do not correspond to any triangulation. Some of their properties are presented. These properties are used to count the forbidden values, which is, finally, exploited in stating another decomposition of the Catalan number.
\end{abstract}

Keywords: Catalan number, Convex polygon triangulation, Binomial coefficient.

\section{Introduction and Preliminaries}

The decomposition of two-dimensional objects into simpler components has a variety of applications in computer graphics as well as in different areas of engineering (robotics, pattern recognition and others mentioned in [3]).

The problem of the convex polygon triangulation assumes dividing a given polygon area by its internal diagonals into the triangles without any gaps and overlaps. This is a classical problem solved so far in several ways.

The number of triangulations of a convex $n$-gon is equal to the $(n-2)$-th Catalan number, i.e.

$$
C_{n-2}=\frac{1}{n-1}\left(\begin{array}{c}
2 n-4 \\
n-2
\end{array}\right)
$$

In [9] we have proposed a recursive convex polygon triangulation algorithm, partially based on the problem solution proposed in [7] where the context-free grammar is used.

Received March 21, 2017; accepted March 13, 2018

2010 Mathematics Subject Classification. Primary 11Y55; Secondary 68R05, 68U05

* The authors were supported in part by Research Project 174013 of the Serbian Ministry of Science and Project "Applying direct methods for digital image restoring" of the Goce Delčev University 
Being inspired by the method described in [7], we have used the following rules generating the arithmetic expression in the reverse Polish notation.

$$
\begin{aligned}
& S \rightarrow S S+ \\
& S \rightarrow b
\end{aligned}
$$

Using the grammar rules (1.2) and (1.3) and the reverse Polish notation (RPN) method (cf. $[8,9,10]$ ) the algorithm for a convex polygon triangulation is suggested in [9]. Also, it is proved that this algorithm works in linear time. Moreover, the correlation between the arithmetic expressions in RPN with triangulations is investigated.

In [9], for every $n \geq 3$, we can consider the mapping $F_{n}: R_{n} \mapsto T_{n}$, whose domain is the set of expressions made by the $n-2$ applications of the rule (1.2) and $n-1$ applications of the rule (1.3), and the range is the set of an $n$-gon triangulations. It is not difficult to verify that some elements from $R_{n}$ could be derived in multiple ways. The unique characterization for each element in $R_{n}$ is given in [9].

Following the usual notations, by $\{+, b\}_{p, q}^{*}$ we denote the subset of the closure set $\{+, b\}^{*}$ consisting of $p$ appearances of the character + and $q$ appearances of the character $b$. In the boundary case, we have $\{+, b\}_{0,0}^{*}=\{\epsilon\}$.

Lemma 1.1. [9] An arbitrary element $r_{n} \in R_{n}$, corresponding to a particular triangulation of an $n$-sided polygon, is uniquely determined by the following two conditions:

(C1) It possesses the form

$$
r_{n}=b b A+, \quad A \in\{+, b\}_{n-3, n-3}^{*},
$$

(C2) Each initial part of the expression $r_{n}$ (the substring of consecutive characters which starts from the first character) must be of the form

$$
\{+, b\}_{p, q}^{*} \quad p<q, \quad p=1, \ldots, n-2, q=1, \ldots, n-1 .
$$

By $P_{n}$ we denote the set of expressions generated by the grammar rules (1.2), (1.3) which satisfy conditions $(C 1)$ and $(C 2)$.

Lemma 1.2. [9] The mapping $F_{n}: P_{n} \mapsto T_{n}$ is well defined, one-to-one and onto for any $n \geq 3$.

In this paper, we assign the unique integer to every arithmetic expression in RPN, derived by rules (1.2) and (1.3), which corresponds to some $n$-gone triangulation. In other words, we have made a bijection between triangulations $T_{n}$ and a subset of integers. We consider these numbers and give some of their properties.

As a consequence, we get an ordering in the set $T_{n}$, different than e.g. the one derived in [5]. In [5] the authors propose the infinite tree of all triangulations, where every tree node is a particular triangulation of an $n$-gon $(n \geqslant 3)$ with a number 
of descendants equal to the degree of the vertex $n$ in range $2, \ldots, n-1$. The descendant triangulations (which are the $(n+1)$-gon triangulations) are derived by "splits" of diagonals $\delta_{i, n}, i \in\{1, \ldots, n-1\}$, incident to the vertex $n$. If these splits are performed in the increasing order of $i$, the unique order among all triangulations (and unique tree of all triangulations) is derived.

In the following section, we define the forbidden combinations (which do not correspond to any element of $T_{n}$ ) and count their number.

There is a variety of recursive definitions of Catalan numbers in literature. Many of them are derived using their combinatorial essence. For example, a well known Touchard's formula (see e.g. [6]) is derived using the generating function for Catalan numbers. Also, Hurtado in [4] gives another recursive definition counting the triangulations by the ears. Stanimirović et al. in [13] introduce Catalan matrix and derive several combinatorial identities with Catalan numbers.

Catalan numbers appear not only in counting the polygon triangulations, but in lattice paths, ballot problem and several tents of other situations which can be found summarized in [14].

They have even a broader application concerning many problems in physics and engineering (see e.g. [2]). Integer sequences themselves are also applicable in variety of areas as queueing theory and probability theory (see [1]) and many others. An interesting application of Catalan numbers sequence in cryptography can be found in $[11]$.

Actually, the present literature on Catalan numbers is far more exhaustive, containing many different formulas for Catalan numbers derived in the variety of approaches and contexts.

Finally, using the combinatorial argument and definition of the forbidden combinations, we give another recursive definition of Catalan numbers.

\section{Arithmetic Expressions and Integer Sequences}

It is obvious that by changing b's with 1's and +'s with 0's we get a unique binary number for every triangulation of the convex polygon.

Denote the integer value corresponding to the $i$ th triangulation from $T_{n}$ by $d_{n}^{i}$. It is of our interest to consider sets

$$
D_{n}=\left\{d_{n}^{i} \mid i=1, \ldots, C_{n-2}\right\}, \quad D=\bigcup_{n \geq 3} D_{n} .
$$

The condition (C1) of Lemma 1.1 imposes that each of those numbers starts with ' 11 ' and ends with ' 0 '. If so, we could consider only the central part of each $d_{n}^{i}$. Denote this binary number by $\left[x_{n}^{i}\right]$ and its value by $x_{n}^{i}$, where $i \in\left\{1, \ldots, C_{n-2}\right\}$ 
corresponds to a particular triangulation. Similarly, we will use the sets

$$
X_{n}=\left\{x_{n}^{i} \mid i=1, \ldots, C_{n-2}\right\}, \quad X=\bigcup_{n \geq 3} X_{n} .
$$

The expressions $P_{n}$ and corresponding numbers for smaller values of $n$ are presented in Table 2.1.

Table 2.1: Sets $P_{n}, D_{n}$ and $X_{n}$.

\begin{tabular}{||l|l|l|r|r||}
\hline \hline$n$ & \multicolumn{1}{|c|}{$P_{n}$} & \multicolumn{1}{|c|}{$\left[p_{n}^{i}\right]$} & $D_{n}$ & $X_{n}$ \\
\hline \hline 3 & $b b+$ & 110 & 6 & 0 \\
\hline 4 & $b b b++$ & 11100 & 28 & 2 \\
& $b b+b+$ & 11010 & 26 & 1 \\
\hline 5 & $b b b b+++$ & 1111000 & 120 & 12 \\
& $b b b+b++$ & 1110100 & 116 & 10 \\
& $b b b++b+$ & 1110010 & 114 & 9 \\
& $b b+b b++$ & 1101100 & 108 & 6 \\
& $b b+b+b+$ & 1101010 & 106 & 5 \\
\hline 6 & $b b b b++++$ & 111110000 & 496 & 56 \\
& $b b b b+b+++$ & 111101000 & 488 & 52 \\
& $b b b b++b++$ & 111100100 & 484 & 50 \\
& $b b b b+++b+$ & 111100010 & 482 & 49 \\
& $b b b+b b+++$ & 111011000 & 472 & 44 \\
& $b b b+b+b++$ & 111010100 & 468 & 42 \\
& $b b b+b++b+$ & 111010010 & 466 & 41 \\
& $b b b++b b++$ & 111001100 & 460 & 38 \\
& $b b b++b+b+$ & 111001010 & 458 & 37 \\
& $b b+b b b+++$ & 110111000 & 440 & 28 \\
& $b b+b b+b++$ & 110110100 & 436 & 26 \\
& $b b+b b++b+$ & 110110010 & 434 & 25 \\
& $b b+b+b b++$ & 110101100 & 428 & 22 \\
& $b b+b+b+b+$ & 110101010 & 426 & 21 \\
\hline \hline
\end{tabular}

It is interesting that the sequence $X$ appears in [12] noted as $A 085183$, derived from the terms of sequence $A 014486$ without their most significant bit (1) and the least significant bit (0). The sequence $A 014486$ represents a list of totally balanced sequences of $2 n$ binary digits written in base 10. A binary expansion of each term from $A 014486$ contains $n$ os and $n$ 1s and reading from left to right (the most significant to the least significant bit), the number of 0s never exceeds the number of $1 \mathrm{~s}[12]$.

Also, note that among famous Stanley's 66 combinatorial interpretations of Catalan numbers ([14]) there is no example identical to the sequence $X_{n}$. Of course, 
being counted by Catalan numbers, a bijection can be established between all of them.

Now, we investigate some basic properties of the sets $D$ and $X$.

Proposition 2.1. The value of the number corresponding to the postfix expression characteristic for particular triangulation of the convex n-gon is

$$
d_{n}^{i}=2\left(2^{2 n-5}+2^{2 n-6}+x_{n}^{i}\right),
$$

where $x_{n}^{i}$ is the changeable part or a shorter binary (decimal) equivalent.

Vice versa

$$
x_{n}^{i}=\frac{d_{n}^{i}-2^{2 n-4}-2^{2 n-5}}{2} .
$$

Proof. It follows immediately from Lemma 1.1.

In order to clarify Table 2.1, in Figure 2.1 we present the relation between the arithmetic expression $b b b b+++$ from $P_{5}$, derived by productions (1.2) and (1.3), and producing the pentagon triangulation (for the algorithm see [9]). The rest of arithmetic expressions and the corresponding triangulations for $n=5$ is presented in Figure 2.2.

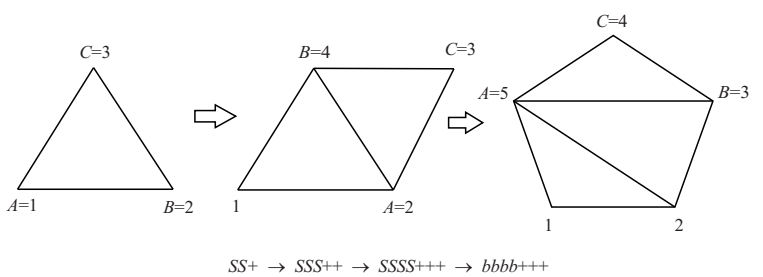

FIG. 2.1: Generating the triangulation corresponding to $b b b b+++$.

It is well known that there is a bijection between the triangulations and binary trees. It is interesting to note that triangulation ordering imposed by sequence $X_{n}$ does not match the usual ordering between the trees corresponding to the particular triangulation (see e.g. [6, Chapter 8]). This can be easily seen in Figure 2.3. In the first column, corresponding arithmetic expression in RPN is presented, then there is the corresponding parsing tree, followed by triangulation and the binary tree for this triangulation. In five rows in Figure 2.3 we have five possible triangulations of a pentagon corresponding to integers $x_{5}^{i}=5,6,9,10,12, i=1,2,3,4,5$ respectively.

Let us give some observations about $\left[x_{n}^{i}\right]$. According to Lemma 1.1, it is obvious that the length of each $\left[x_{n}^{i}\right]$ is equal to $\left|\left[x_{n}^{i}\right]\right|=2 n-6$ and $\left[x_{n}^{i}\right] \in\{0,1\}_{n-3, n-3}^{*}$. Moreover, $\left[x_{n}^{i}\right]$ can not start with more than one ' 0 ' and can not end with more than one '1'. So, it must have one of the forms $\left[y_{1}(n)\right], \ldots,\left[y_{9}(n)\right]$ presented in Table 2.2. 


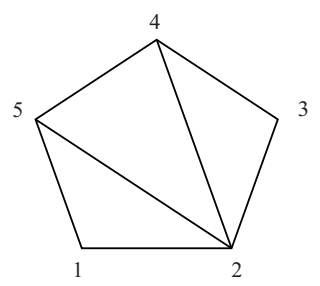

$b b b+b++$

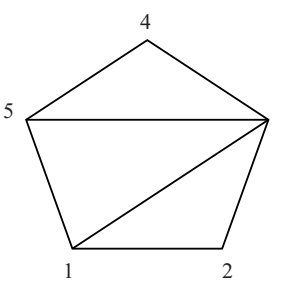

$b b+b+b+$

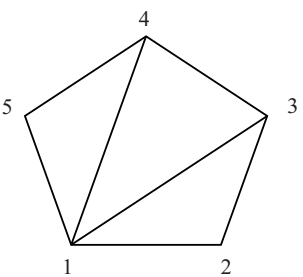

$b b b++b+$

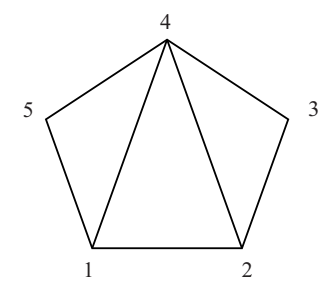

$b b+b b++$

Fig. 2.2: Illustration of the rest from $T_{5}$ and $P_{5}$.

In Table 2.2

$$
\begin{aligned}
& {[\alpha] \in\{0,1\}_{n-6, n-4}^{*}} \\
& {[\beta] \in\{0,1\}_{n-5, n-5}^{*}} \\
& {[\gamma] \in\{0,1\}_{n-4, n-6}^{*} .}
\end{aligned}
$$

Note that some of $[\alpha],[\beta],[\gamma]$ type strings are proper (correspond to particular polygon triangulations) while some of them are not. These strings are proper under conditions described bellow.

The $[\alpha]$ type string can be found only within the contexts

$$
1101[\alpha] 000,1110[\alpha] 000
$$

and, according to the conditions of Lemma 1.1, start of proper $[\alpha]$ should satisfy

$$
[\alpha]_{p s} \in\{0,1\}_{p, q}^{*}, \quad p \leq q+1, \quad p \in\{0, \ldots, n-6\} ; q \in\{0, \ldots, n-4\} .
$$

The substring of $[\beta]$ type has to be considered in two subtypes: $\left[\beta^{\prime}\right]$ and $\left[\beta^{\prime \prime}\right]$. The subtype $\left[\beta^{\prime}\right]$ can be found in the contexts

$$
1101\left[\beta^{\prime}\right] 010,1101\left[\beta^{\prime}\right] 100,1110\left[\beta^{\prime}\right] 010,1110\left[\beta^{\prime}\right] 100,
$$

and, according to Lemma 1.1, every start of proper $\left[\beta^{\prime}\right]$ should satisfy

$$
\left[\beta^{\prime}\right]_{p s} \in\{0,1\}_{p, q}^{*}, \quad p \leqslant q+1, \quad p \in\{0, \ldots, n-5\} ; q \in\{0, \ldots, n-5\} .
$$


Table 2.2: String types.

\begin{tabular}{||c|ccccc||}
\hline \hline weights & $2 n-7$ & $2 n-8$ & $\ldots$ & 1 & 0 \\
\hline$\left[y_{1}(n)\right]$ & 0 & 1 & {$[\alpha]$} & 0 & 0 \\
{$\left[y_{2}(n)\right]$} & 0 & 1 & {$[\beta]$} & 0 & 1 \\
{$\left[y_{3}(n)\right]$} & 0 & 1 & {$[\beta]$} & 1 & 0 \\
{$\left[y_{4}(n)\right]$} & 1 & 0 & {$[\alpha]$} & 0 & 0 \\
{$\left[y_{5}(n)\right]$} & 1 & 0 & {$[\beta]$} & 0 & 1 \\
{$\left[y_{6}(n)\right]$} & 1 & 0 & {$[\beta]$} & 1 & 0 \\
{$\left[y_{7}(n)\right]$} & 1 & 1 & {$[\beta]$} & 0 & 0 \\
{$\left[y_{8}(n)\right]$} & 1 & 1 & {$[\gamma]$} & 0 & 1 \\
{$\left[y_{9}(n)\right]$} & 1 & 1 & {$[\gamma]$} & 1 & 0 \\
\hline \hline
\end{tabular}

The subtype $\left[\beta^{\prime \prime}\right]$ should be found within context

$$
1111\left[\beta^{\prime \prime}\right] 000
$$

and every start of proper $\left[\beta^{\prime \prime}\right]$ should satisfy

$$
\left[\beta^{\prime \prime}\right]_{p s} \in\{0,1\}_{p, q}^{*}, \quad p \leq q+3, \quad p \in\{0, \ldots, n-5\} ; q \in\{0, \ldots, n-5\} .
$$

Because of the fact that $[\gamma]$ type strings can be found only within the contexts

$$
1111[\gamma] 010,1111[\gamma] 100
$$

and the conditions of Lemma 1.1, it is clear that every start of proper $[\gamma]$ should satisfy

(2.4) $[\gamma]_{p s} \in\{0,1\}_{p, q}^{*}, \quad p \leqslant q+3, \quad p \in\{0, \ldots, n-4\} ; q \in\{0, \ldots, n-6\}$.

The number of the bits in $[\alpha],[\beta]$ and $[\gamma]$ is

$$
|[\alpha]|=|[\beta]|=|[\gamma]|=2 n-10
$$

and values of the above binary numbers $\left[y_{i}(n)\right]$ are correspondingly:

$$
\begin{aligned}
& y_{1}(n)=2^{2 n-8}+4 \alpha \\
& y_{2}(n)=2^{2 n-8}+4 \beta+1 \\
& y_{3}(n)=2^{2 n-8}+4 \beta+2 \\
& y_{4}(n)=2^{2 n-7}+4 \alpha \\
& y_{5}(n)=2^{2 n-7}+4 \beta+1 \\
& y_{6}(n)=2^{2 n-7}+4 \beta+2 \\
& y_{7}(n)=2^{2 n-7}+2^{2 n-8}+4 \beta \\
& y_{8}(n)=2^{2 n-7}+2^{2 n-8}+4 \gamma+1 \\
& y_{9}(n)=2^{2 n-7}+2^{2 n-8}+4 \gamma+2
\end{aligned}
$$


Proposition 2.2. The minimal value among the values (2.5)-(2.13) is

$$
y_{\min }(n)=\frac{4^{n-3}-1}{3}, \quad n \geq 4 .
$$

Proof. It is not difficult to observe that the minimal value $y_{\min }(n)$ must have the form $01\left[\beta_{\min }\right] 01$, where $\left[\beta_{\min }\right]$ corresponds to the minimal value in the context $01[\beta] 01$. Note that $\beta_{\min }$ is not the minimal $\beta$ in general.

As a consequence of Lema 1.1 and its condition $(\mathrm{C} 2)$, every beginning of $\beta$, $\beta_{s} \in\{0,1\}_{p, q}^{*}$, satisfies the condition $p \leqslant q+1$. The $\beta_{\text {min }}$ for an $n$-gon (denoted as $\left.\beta_{\min }(n)\right)$ is of the following form:

$$
\begin{aligned}
\beta_{\min }(n) & =\epsilon, \quad 3 \leq n \leq 5 \\
\beta_{\min }(6) & =01=1_{10}, \\
\beta_{\min }(7) & =0101=5_{10}, \\
\beta_{\min }(n>7) & =\overbrace{\overline{01}}^{n-5}=\sum_{k=1}^{n-5} 2^{2(k-1)} .
\end{aligned}
$$

Therefore

$$
\begin{aligned}
y_{\min }(n) & =2^{2 n-8}+4 \sum_{k=1}^{n-5} 2^{2(k-1)}+1 \\
& =2^{2 n-8}+4 \frac{2^{2 n-10}-1}{3}+1 \\
& =4^{n-4}+4 \frac{4^{n-5}-1}{3}+1 \\
& =\frac{3 \cdot 4^{n-4}+4 \cdot 4^{n-5}-4+3}{3} \\
& =\frac{4^{n-3}-1}{3} .
\end{aligned}
$$

Proposition 2.3. The maximal value among the values (2.5)-(2.13) is

$$
y_{\max }(n)=2^{n-3}\left(2^{n-3}-1\right) .
$$

Proof. It is straightforward to conclude that the maximal value must have the form $11\left[\beta_{\max }\right] 00$, where $\left[\beta_{\max }\right]$ corresponds to the maximal value in the context $11[\beta] 00$, since it starts with two 1's and $\beta$ has more 1's than $\gamma$.

The maximal $\beta$ (which is allowed by Lemma 1.1) is

$$
\left[\beta_{\max }\right]=\underbrace{1 \ldots 1}_{n-5} \underbrace{0 \ldots 0}_{n-5}=1^{n-5} 0^{n-5} .
$$


The corresponding value is

$$
\beta_{\max }=2^{n-5} \sum_{k=0}^{n-6} 2^{k}=2^{n-5}\left(2^{n-5}-1\right)
$$

Therefore, using

$$
y_{\max }(n)=2^{2 n-7}+2^{2 n-8}+4 \beta_{\max }
$$

and the elementary algebraic operations the proof can be completed.

Proposition 2.4. The following inequality is satisfied for $n \geq 3$ :

$$
y_{\min }(n+1)>y_{\max }(n) .
$$

Proof. According to Propositions 2.2 and 2.3

$$
\begin{aligned}
y_{\min }(n+1)-y_{\max }(n) & =\frac{4^{n-2}-1}{3}-2^{n-3}\left(2^{n-3}-1\right) \\
& =\frac{a^{2}+3 a-1}{3},
\end{aligned}
$$

where $a=2^{n-3}$. As $n \geq 4$ and $a \geq 2$, it is not difficult to verify that the quadratic inequality

$$
a^{2}+3 a-1>0
$$

is satisfied.

As we have already seen, some combinations are forbidden (do not correspond to any $n$-gon triangulation). Later, we count these forbidden combinations. In accordance to the previous results we state the next definition.

Definition 2.1. The forbidden starts are defined in the following way:

(a) By the forbidden start of $[\alpha]$ we consider

$$
[\alpha]_{f s} \in\{0,1\}_{p, q}^{*}, p>q+1,
$$

where all its starts with length less than $p+q$ are proper.

(b) By the forbidden start of $\left[\beta^{\prime}\right]$ we consider

$$
\left[\beta^{\prime}\right]_{f s} \in\{0,1\}_{p, q}^{*}, p>q+1,
$$

where all its starts with length less than $p+q$ are proper.

(c) The forbidden start of $\left[\beta^{\prime \prime}\right]$ is given by

$$
\left[\beta^{\prime \prime}\right]_{f s} \in\{0,1\}_{p, q}^{*}, p>q+3,
$$

where all its starts with length less than $p+q$ are proper. 
(d) By the forbidden start of $[\gamma]$ we consider

$$
[\gamma]_{f s} \in\{0,1\}_{p, q}^{*}, p>q+3,
$$

where all its starts with length less than $p+q$ are proper.

Proposition 2.5. Any combination having the forbidden start is also forbidden.

Remark 2.1. The forbidden combination is not a proper string $\{0,1\}_{n-3, n-3}^{*}$ which corresponds to some n-gon triangulation. The purpose of the forbidden start notion is to count the non-proper combinations which do not correspond to any triangulation. If we recognize a forbidden start, then any pattern of bits following it makes a certain number of forbidden combinations. This will be used later in Theorems 2.1 and 2.2.

The following lemmas hold.

Lemma 2.1. If there exists a forbidden start in $[\alpha],[\beta]$ or $[\gamma]$ it must contain an even number of characters.

Proof. According to (2.1) and (2.2), the shortest forbidden start for $[\alpha]$ and $\left[\beta^{\prime}\right]$ is '00', where $p+q=2$, an even integer. Similarly, according to (2.3) and (2.4), the shortest forbidden start for $\left[\beta^{\prime \prime}\right]$ and $[\gamma]$ is '0000', in which case holds $p+q=4$.

The case $p+q=2 q+1$ is the limit case for allowed start in (2.1) and (2.2). The forbidden start can grow from it by adding one zero at the end, making the length of this forbidden start an even number $p+1+q=2 q+2$.

For all allowed starts of $\left[\beta^{\prime \prime}\right]$ and $[\gamma]$ holds $p \leq q+3$. The forbidden start can be made by appending one zero only for the case $p=q+3$. Therefore, $p+q=$ $2 q+3$ must be satisfied again. In this case, the forbidden start has the even length $p+1+q=2 q+4$.

Lemma 2.2. The following holds:

(a) If we have a forbidden start $2 k$ characters long in $[\alpha]$ or $\left[\beta^{\prime}\right]$, then it contains exactly $k-11 s$.

(b) If we have a forbidden start $2 k$ characters long in $\left[\beta^{\prime \prime}\right]$ or $[\gamma]$, then it contains exactly $k-21 s$.

Proof. (a) Let us consider firstly the $[\alpha]$ type case. As $p+q=2 k$ and (2.) are satisfied, we have $p=q+2$ which implies $q+2+q=2 k$ and the proof is completed.

The rest of the proof could be done in a similar way. 
Remark 2.2. It is easy to observe that the shortest forbidden start '00' for $[\alpha]$ occurs at $n=8$. Regarding $[\alpha] \in\{0,1\}_{n-6, n-4}^{*}$, this forbidden start can be found in the combinations $1101 \underline{001111000}$ or $1110 \underline{001111000}$.

Also, for $\left[\beta^{\prime}\right]$ the shortest forbidden start '00' occurs at $n=7$ in 11010011010 , $1101 \underline{0011} 100,1110 \underline{0011} 010$ or $1110 \underline{0011100 .}$

In the case of $\left[\beta^{\prime \prime}\right]$ the shortest forbidden start is 0000 ', at $n=9$ in combination $1111 \underline{00001111000 .}$

The shortest forbidden start '0000' for $[\gamma]$ occurs at $n=8$ in

$$
1111 \underline{000011} 010
$$

or

\section{$1111 \underline{000011} 100$.}

Theorem 2.1. The number of forbidden combinations for $[\alpha]$ is

$$
n_{\alpha_{f s}}(n)=\left(\begin{array}{c}
2 n-10 \\
n-8
\end{array}\right), \quad n \geq 8 .
$$

The number of forbidden combinations for $\left[\beta^{\prime}\right]$ is

$$
n_{\beta_{f s}^{\prime}}(n)=\left(\begin{array}{c}
2 n-10 \\
n-7
\end{array}\right), \quad n \geq 7 .
$$

The number of forbidden combinations for $\left[\beta^{\prime \prime}\right]$ and $[\gamma]$ is

$$
n_{\beta_{f s}^{\prime \prime}}(n)=\left(\begin{array}{c}
2 n-10 \\
n-9
\end{array}\right), n \geq 9,
$$

and

$$
n_{\gamma_{f s}}(n)=\left(\begin{array}{c}
2 n-10 \\
n-8
\end{array}\right), n \geq 8,
$$

respectively.

Proof. As $[\alpha]_{f s} \in\{0,1\}_{p, q}^{*}, p>q+1$, and $[\alpha] \in\{0,1\}_{n-6, n-4}^{*}$, we actually want to count all paths from $(0,0)$ to $(n-6, n-4)$ touching the line $p=q+2$. Using the reflection principle, this is the same number as the number of paths from $(2,-2)$ to $(n-6, n-4)$. This yields

$$
n_{\alpha_{f s}}(n)=\left(\begin{array}{c}
n-6+n-4 \\
n-6-2
\end{array}\right)=\left(\begin{array}{c}
2 n-10 \\
n-8
\end{array}\right) .
$$

In a similar way, we can complete the rest of the proof. Note that for $\left[\beta^{\prime}\right]_{f s} \in$ $\{0,1\}_{p, q}^{*}, p>q+1$ and $[\beta] \in\{0,1\}_{n-5, n-5}^{*}$ we need to observe count of paths from 
$(2,-2)$ to $(n-5, n-5)$, while for $\left[\beta^{\prime \prime}\right]_{f s} \in\{0,1\}_{p, q}^{*}, p>q+3$, we observe line $p=q+4$ and paths from $(4,-4)$ to $(n-5, n-5)$.

For $[\gamma]_{f s} \in\{0,1\}_{p, q}^{*}, p>q+3$, and $[\gamma] \in\{0,1\}_{n-4, n-6}^{*}$ we need to observe line $p=q+4$ and paths from $(4,-4)$ to $(n-4, n-6)$.

In the next theorem, we state another way to express Catalan number.

\section{Theorem 2.2.}

$$
\begin{aligned}
C_{n-2}=4\left(\begin{array}{c}
2 n-10 \\
n-6
\end{array}\right) & +5\left(\begin{array}{c}
2 n-10 \\
n-5
\end{array}\right)-4\left(\begin{array}{c}
2 n-10 \\
n-8
\end{array}\right) \\
& -4\left(\begin{array}{c}
2 n-10 \\
n-7
\end{array}\right)-\left(\begin{array}{c}
2 n-10 \\
n-9
\end{array}\right), n \geq 9 .
\end{aligned}
$$

Proof. The proof could be derived in the combinatorial way. The overall number of possible combinations in the case of $[\alpha]$ and $[\gamma]$ type strings from Table 2.2 is $4\left(\begin{array}{c}2 n-10 \\ n-6\end{array}\right)$, while the number of possible combinations in the case of $[\beta]$ is $5\left(\begin{array}{c}2 n-10 \\ n-5\end{array}\right)$.

Theorem 2.1 counts all forbidden combinations. The overall number of possible combinations diminished by the number of the forbidden combinations results in the number of the allowed combinations, which is equal to $C_{n-2}$.

Corollary 1. The following holds

$$
\begin{aligned}
C_{n-2} & =8 \frac{8 n^{3}-84 n^{2}+286 n-315}{(n-1)(n-2)(n-3)(n-4)}\left(\begin{array}{c}
2 n-10 \\
n-5
\end{array}\right) \\
& =8 \frac{8 n^{3}-84 n^{2}+286 n-315}{(n-1)(n-2)(n-3)} C_{n-5} .
\end{aligned}
$$

Proof. Using Cardan's rule of succession

$$
\left(\begin{array}{l}
n \\
r
\end{array}\right)=\frac{n-r+1}{r}\left(\begin{array}{c}
n \\
r-1
\end{array}\right)
$$

each binomial coefficient from (2.14) can be reduced on the same type, e.g. $\left(\begin{array}{c}2 n-10 \\ n-5\end{array}\right)$. After some algebraic transformations we get the result. 


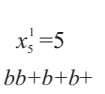<smiles>[2H][13CH][13CH][13CH]C[18O]</smiles>
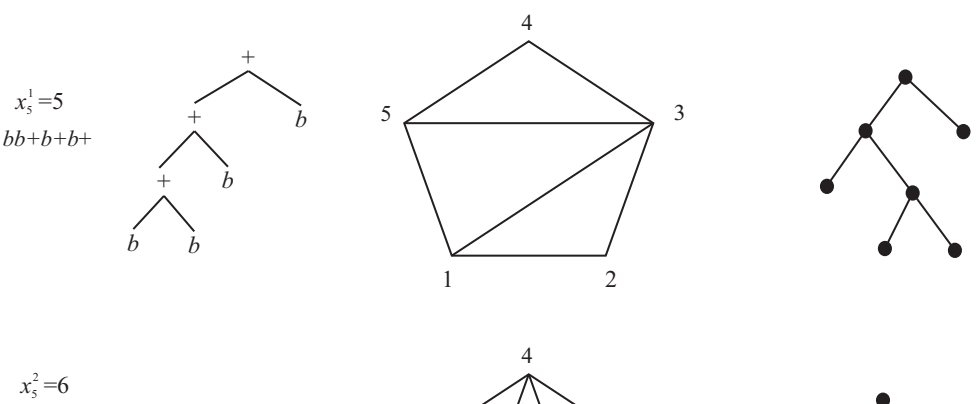

$b b+b b++$<smiles>[2H][C+][C+][18O]</smiles>
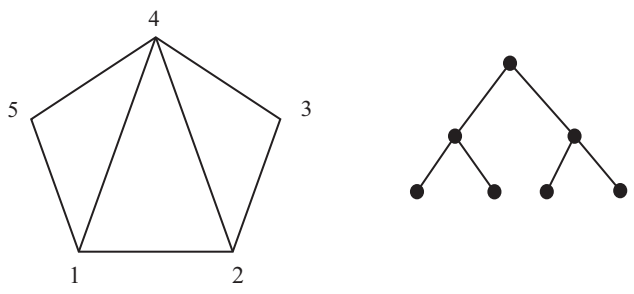

$x_{5}^{3}=9$
$b b++b+b+2$<smiles>C[C+]PC(C)P</smiles>
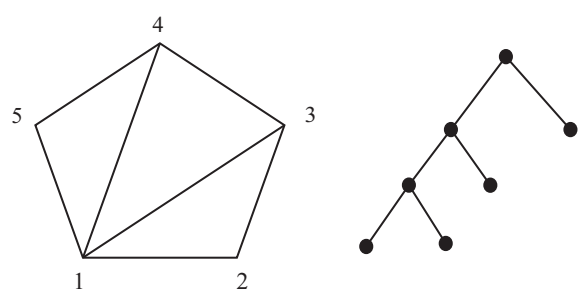

$x_{5}^{4}=10$

$b b b+b++$

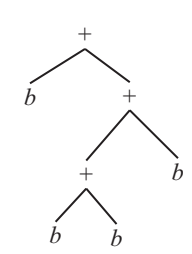
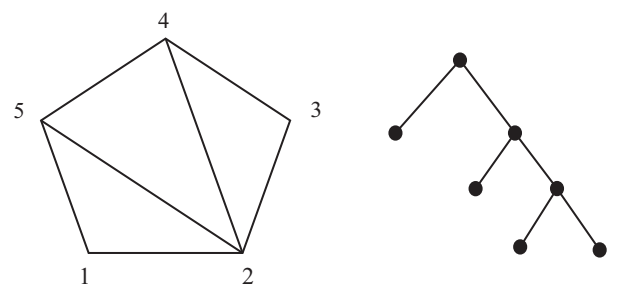

$x_{5}^{5}=12$
$b b b b+++$
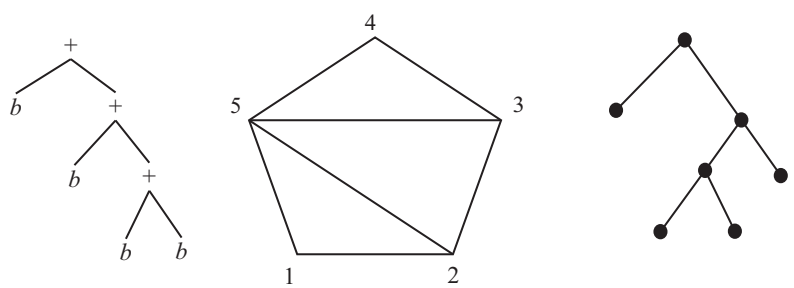

FIG. 2.3: Illustrations of arithmetic expressions, expression trees, triangulations and binary trees. 


\section{Conclusion}

We have made the presentation of the convex polygon triangulations in the form of integers. This imposes one of the triangulation ordering different from e.g. the one derived in [5].

Moreover, the boundaries for these integers are found in terms of polygon vertices number $n$. The forbidden patterns in the binary presentation of these integers are counted. Another way to express Catalan number via binomial coefficients is presented.

\section{Acknowledgments}

The authors wish to express their gratitude to prof. Dragan Stevanović who read the draft of this paper and gave a number of useful comments and suggestions. His effort provides a considerable contribution to the quality of this paper.

\section{R E F E R E N C E S}

1. J. Abate, W. Whitt, Integer sequences from queueing theory, Journal of Integer Sequences, 13 (2010), 21p.

2. J. Abate, W. Whitt, Brownian motion and the generalized Catalan numbers, Journal of Integer Sequences, 14 (2011), 15p.

3. B. Chazelle, L. Palios, Decomposition algorithms in geometry, Algebraic Geometry and its Application (ed. Ch. L. Bajaj) 27, (1994), 419-447.

4. F. Hurtado, M. Noy, Ears of triangulations and Catalan numbers, Discrete Mathematics, 149 (1996), 319-324.

5. F. Hurtado, M. Noy, Graph of triangulations of a convex polygon and tree of triangulations, Comput. Geom., 13 (1999), 179-188.

6. T. Koshy, Catalan numbers with applications, Oxford University Press, Oxford, New York, 2009.

7. M. Kross, A. Lentin, Notions sur les grammaries formelles, Gauthier-Villars, 1967.

8. P. V. Krtolica, P. S. Stanimirović, On Some properties of Reverse Polish Notation, FILOMAT, 13 (1999), 157-172.

9. P. V. Krtolica, P. S. Stanimirović, R. Stanojević, Reverse Polish notation in constructing the algorithm for polygon triangulation, FILOMAT, 15 (2001), 25-33.

10. P. V. Krtolica, P. S. Stanimirović, Reverse Polish notation method, Int. J. Comput. Math., 81(3) (2004), 273-284.

11. D. Sravana Kumar, CH. Suneetha, A. Chandrasekhar, Novel encryption schemes based on Catalan numbers, International Journal of Engineering Research and Applications (IJERA), 2(2) (2012), 161-166.

12. The On-Line Encyclopedia of Integer Sequences (OEIS). http://oeis.org/Seis.html. 
13. S. Stanimirović, P. Stanimirović, M. Miladinović, A. Ilić, Catalan matrix and related combinatorial identities, Appl. Math. Comput., 215 (2009), 796-805.

14. R. P. Stanley, Enumerative Combinatorics, vol. 2, Cambridge University Press, Cambridge, 1999 .

Predrag Krtolica

University of Niš, Faculty of Science and Mathematics Department of Computer Science

Višegradska 33, 18000 Niš, Serbia

krca@pmf.ni.ac.rs

Predrag Stanimirović

University of Niš, Faculty of Science and Mathematics Department of Computer Science

Višegradska 33, 18000 Niš, Serbia

pecko@pmf.ni.ac.rs

Igor Stojanović

Goce Delčev University, Faculty of Computer Science

Goce Delčev 89, 2000 Štip, Macedonia

igor.stojanovik@ugd.edu.mk 